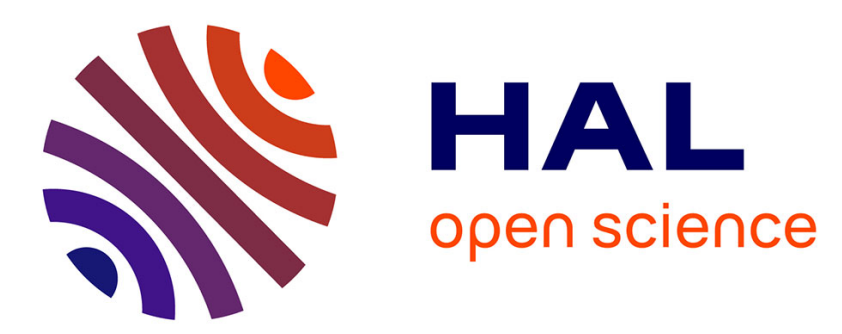

\title{
Chyme reinfusion or enteroclysis in nutrition of patients with temporary double enterostomy or enterocutaneous fistula
}

Ronan Thibault, Denis Picot

\section{- To cite this version:}

Ronan Thibault, Denis Picot. Chyme reinfusion or enteroclysis in nutrition of patients with temporary double enterostomy or enterocutaneous fistula. Current Opinion in Clinical Nutrition and Metabolic Care, 2016, 19 (5), pp.382-387. 10.1097/MCO.0000000000000304 . hal-01390778

HAL Id: hal-01390778

https://hal-univ-rennes1.archives-ouvertes.fr/hal-01390778

Submitted on 2 Nov 2016

HAL is a multi-disciplinary open access archive for the deposit and dissemination of scientific research documents, whether they are published or not. The documents may come from teaching and research institutions in France or abroad, or from public or private research centers.
L'archive ouverte pluridisciplinaire HAL, est destinée au dépôt et à la diffusion de documents scientifiques de niveau recherche, publiés ou non, émanant des établissements d'enseignement et de recherche français ou étrangers, des laboratoires publics ou privés. 
1 Chyme reinfusion or enteroclysis in nutrition of patients with temporary double

2 enterostomy or enterocutaneous fistula

3

4 Ronan Thibault, ${ }^{\mathrm{a}}$ Denis Picot $^{\mathrm{b}}$

5

$6 \quad{ }^{a}$ Nutrition unit, Department of Endocrinology, Diabetology and Nutrition, Home parenteral

7 nutrition centre, CHU Rennes, Université de Rennes 1, INSERM U991, Rennes, F-35000,

8 France

$9 \quad$ b Department of Nutritional and Digestive Rehabilitation, Clinique Saint Yves, Rennes, F10 35044, France

11

12 Corresponding author:

13 Prof. Ronan Thibault, $\mathrm{MD}, \mathrm{PhD}$

14 Unité de Nutrition

15 CHU Rennes - Pontchaillou

16 2, rue Henri Le Guilloux

$17 \quad 35000$ Rennes

18 France

19 Phone +33299289646

$20 \quad$ Fax +33299289647

21 E-mail ronan.thibault@chu-rennes.fr 


\section{Abstract (196 words)}

\section{Purpose of review}

Patients with double temporary enterostomy or enterocutaneous fistula (ECF) may suffer from intestinal failure (IF). Parenteral nutrition (PN) is the gold standard treatment until surgical reestablishment of intestinal continuity, but serious complications may arise. Chyme reinfusion (CR) or enteroclysis are indicated.

\section{Recent findings}

CR corrects the IF by restoring intestinal absorption, allowing PN weaning in $91 \%$ of patients. CR contributes to improve nutritional status and reduce plasma liver tests abnormalities. CR is feasible at home without any serious complications in selected patients. Mechanisms underlying CR effectiveness on intestinal function, such as restoration of ileal brake, are suggested but most remain to be demonstrated. When the downstream small bowel is exposed, enteroclysis of enteral nutrition or hydration could be helpful to reduce PN needs, or in case of insufficient food intake during CR.

\section{Summary}

CR or enteroclysis are less expensive, safe, and easy-to-use nutrition support techniques, that may allow reducing PN-related healthcare costs. The latter remains to be demonstrated in the setting of a prospective randomized controlled trial. This review may contribute to improve the awareness of intensivists, digestive surgeons and gastroenterologists involved in IF management to spread the use of CR or enteroclysis.

Keywords: intestinal failure; fistuloclysis; gastrointestinal (GI) surgery; EN; parEN 


\section{Introduction}

In the course of an intestinal surgery procedure, several clinical situations lead the surgeon to undertake a double temporary enterostomy (small bowel resection, peritonitis, fistulae, anastomosis protection...) or could be complicated of enterocutaneous fistula (ECF) (peritonitis, anastomosis leakage, digestive adherences...). These conditions could constitute a type 1 short bowel syndrome and are often complicated with intestinal failure (IF), especially when the stoma output is equal or higher than $1500 \mathrm{ml} / 24 \mathrm{~h}$. These lead to serious complications resulting in hospital readmissions, such as acute or chronic dehydration, renal failure, electrolyte disturbances, micronutrients and mineral deficiencies, and malnutrition, thus increasing healthcare-related costs and affecting patients' quality of life [1]. IF was recently defined by the European Society for Clinical Nutrition and Metabolism (ESPEN) as "the reduction of gut function below the minimum necessary for the absorption of macronutrients and/or water and electrolytes, such that intravenous supplementation is required to maintain health and/or growth » [2]. In case of temporary double enterostomy or ECF, the IF is type 2, and defines as a prolonged acute condition, often in metabolically unstable patients, requiring complex multi-disciplinary care and intravenous supplementation over periods of weeks or months [2]. At this time, the current gold standard therapy indicated until the surgical reestablishment of digestive continuity (SIRC), with a mean duration of 3-6 months, is home parenteral nutrition (HPN) [3]. However, HPN has its own morbidity and, in the absence of expertise, the risks of infectious, hepatic dysfunction, mechanical and metabolic complications are increased [1,3]. Therefore, the availabilities of low cost, safer, and easy-to-use nutrition support techniques could be of high added value in these type 2 IF patients. Chyme reinfusion (CR) [4-9] and enteroclysis [5,6,10] could be these techniques. The scope of this review is to describe the technical principles of CR and enteroclysis, to give practical details for their use, review their clinical benefits in clinical practice, and, due to the lack of data, to hypothesize the mechanisms related to their clinical benefits. 


\section{Definitions}

73 The interruption of the small bowel by a double enterostomy or an ECF separates the small

74

bowel into an upstream afferent segment and a downstream efferent segment (Figure 1).

Enteroclysis is an enteral nutrition (EN) technique consisting in the administration of EN or hydration solutions in the downstream efferent small bowel through efferent enterostomy or ECF exposed to the abdomen wall, chyme from the afferent small bowel being thrown out. The term "fistuloclysis" [2,6,10] wrongly used as a synonym of "enteroclysis” should be abandoned since: it is medically inappropriate, referring to a technique of fistula irrigation or washing and not to an EN technique which could be only delivered in the intestine through the fistula, but not in the fistula; it is linguistically inappropriate: one Latina prefix followed by one Greek suffix. CR [4] (or re-feeding enteroclysis [2,5] or succus entericus reinfusion $[8,9])$ is an EN technique which artificially re-establishes the small bowel continuity by an extra-corporeal circuit of the chyme and mimics the definitive gastro-intestinal function; the chyme, composed of digestive secretions and nutrients from oral food and/or tube feeding, is collected from the afferent small bowel, and reinfused via the enterostomy or the ECF into the efferent diverted small bowel segment. CR could be, at best, in our experience, continuous, through a portable or not automated pump, or, as reported by others [5], sequential by manual chyme decanting.

\section{The principle of CR with auto-regulated pumps}

Although first described in 1977 by Etienne Levy, and recently suggested as an alternative therapy in IF patients [2], CR is rarely used, under recognized, and not endorsed by most health insurances. This is partly due to the fact that the materials used for CR were not specifically dedicated to the technique. Indeed, in most centres where it is used, CR is performed by diverting from their first use EN pumps and tubulures, or dialysis material... 
97 Usually, and we think that this technique must be abandoned, chyme was collected in a bag

98 thanks to gravity, sometimes refrigerated, often sieved to remove the largest food particles.

99 Chyme is then transferred every 3-4 hours in an EN bag to be reinfused with an auto-

100 regulated EN pump into the downstream efferent small bowel. Others directly reinfused the

101 chyme with a syringe. All these handlings were associated with uncomfortable odours and

102 dirts making the CR technique very unpopular. Spreading the use of auto-regulated pumps

103 dedicated to CR would help to increase CR acceptation and use in IF-specialized centres. At

104 Clinique Saint Yves, Rennes, France, we perform continuous CR using the Entéromate® II system (Labodial, Les Clayes Sous Bois, France), marketed since 1998 (Figure 2).

Entéromate ${ }^{\circledR}$ II auto-regulates continuous CR without any adjustment or nurse's intervention,

107 and no uncomfortable odour. The dead space volume of the extra-corporeal circuit is lower

108 than $50 \mathrm{ml}$ and does not cause any volemic deprivation. The tubulures are closed and prevent

109 from outside infectious contamination. The automaton has two peristaltic pumps. One pump

110 works permanently and aspirates the jejunal effluent toward a $30 \mathrm{~mL}$ disposable plastic

111 container, so that the upper stoma pouch is always empty. The weight of the container is

112 continuously and electronically monitored. When the minimal volume of approximately $10 \mathrm{ml}$

113 is exceeded, the second pump starts and the contents are infused into the diverted downstream

114 small bowel until the return to minimal volume. The downstream small bowel is intubated

115 through the efferent enterostomy or ECF with a simple lumen polyurethane naso-gastric tube

116 ch 14-16, Levine-typed, without balloon, into the first 15-20 centimetres of the small bowel

117 (Figure 1). We advise that polyurethane naso-gastric tubes must be preferred to Foley’s

118 because of their higher internal diameter (for a given "ch" calibre) and the absence of balloon,

119 that could injury the small bowel when too much inflated. Ideally a radiologic opacification

120 with water-soluble contrast agents checks the tube position and controls the anatomy and the

121 length of the downstream small bowel until the colon. 


\section{Indications of CR}

In our experience, the patients eligible for CR fulfil the following criteria: IF defined as a theoretical indication to PN, plus a stoma output nihil per mouth of at least $1200 \mathrm{ml} / 24 \mathrm{~h}$; existence of a double enterostomy or at least two orifices of ECF visible on the abdominal wall; theoretical temporary nature of the stoma or ECF in the expectancy of SIRC; presence of efferent small bowel between the stoma and the colon, or a terminal ileostomy; absence of obstruction of digestive fistula between the mouth and the afferent stoma, and in the efferent intestinal tract; ability to catheterize the efferent stoma with a feeding tube on more than 15 cm; absence of progressive peritoneal carcinosis; age >17 years; full agreement of the patient to carry out CR and accept the food constraints of ingesting smooth puree meals.

\section{CR and enteroclysis in daily practice}

CR and enteroclysis should be integrated in a global approach of intestinal rehabilitation. Patients require complex management of opened abdominal wounds, high intestinal outputs and need a multi-disciplinary nutrition team, including specially trained nurses and nutritionist gastroenterologists or surgeons. During the two days before CR initiation, enteroclysis is initiating by instilling one liter of oral rehydration solution, together with laxatives in case of fecal residues or fecaloma in the colon. At the same time, anti-motility drugs, e.g. loperamide, are stopped to prevent ileus. Antispasmodic agents could be useful in case of abdominal pain, and cholestyramine is given by enteroclysis in the event of diarrhea during the first days. In case of persisting diarrhea, loperamide is used. Antisecretory gastric drugs are used in all patients before and during CR. Octreotide is never used. Once the patient has been adequately trained and is capable of correctly adjusting the rate of reinfusion, portable non-autoregulated Enteromate Mobile ${ }^{\circledR}$ pump (Labodial, Les Clayes Sous Bois, France), marketed since 2010, is used secondly to give autonomy to the patient. This pump is autonomous thanks to batteries. During CR, to avoid tubes obstruction, patients are 
149 mandatorily orally fed ad libitum with smooth puree meals. In case of insufficient food or

150 hydration intake during CR, EN and additional hydration solutions could be administered

151 classically through a nasogastric feeding tube, gastrostomy or jejunostomy, or, in some cases,

152

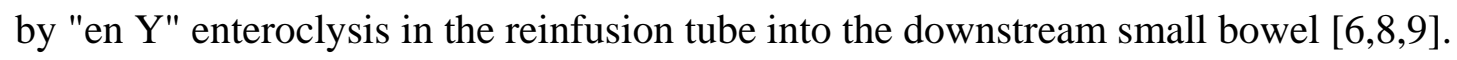

153

\section{Clinical benefits of $\mathbf{C R}$ and enteroclysis}

155 Only a few studies have reported the beneficial effects of CR or enteroclysis in IF patients

156 with temporary double enterostomy or ECF. Only one [4] was published within 18 months

157 and none were prospective randomized controlled trials. These studies are monocentric case

158 series, having included only a low number of patients, and did not report post-SIRC clinical

159 outcomes. In adult patients, CR could restore intestinal absorptive capacities [4,7]. This could

160 allow PN discontinuation a few days after its initiation [4,5], and the improvement of

161 frequently observed liver tests abnormalities [4]. Plasma liver tests improvement seems

162 greater in patients treated by enteroclysis coupled with CR than by enteroclysis alone [6]. In

163 neonates and premature infants with double enterostomies, CR reduces PN dependence and

164 and corrects plasma liver tests abnormalities [9]. CR improved nutritional status [4,6,7]. The

165 larger monocentric (Clinique Saint Yves, Rennes, France) prospective cohort assessing the

166 efficacy of CR in 212 patients with a temporary double enterostomy (86\% of patients) or ECF

167 (14\%) waiting for SIRC is under publication and confirms these findings, and suggests the

168 feasibility of home CR [4]. Double enterostomy or ECF were mainly due to peritonitis (44\%)

169 and cancer (34\%). CR corrects the IF by restoring intestinal absorption: reduction in intestinal

170 losses by 85\%, strong improvement in nitrogen and fat digestive absorption coefficients, and

171 strong reduction in the proportion of patients with plasma citrulline $<20 \mu \mathrm{mol} / \mathrm{l}$ [4]. As a

172 result, PN and/or IV hydration could be stopped in 91\% of patients, within a

173 median \pm interquartiles (IQ) of $2 \pm 9$ days after CR initiation [4], or in $100 \%$ of cases within

17420 days in the little case series of 20 patients reported by Coetzee et al [5]). In addition, 
175 nutritional status improved. With CR, the number of patients who had one or several plasma

176 liver tests abnormalities decreased from 87 to 51\% $(\mathrm{P}<0.001)$ [4].

\section{Complications and side effects of CR}

179 The management of CR-related complications and side effects requires dedicated healthcare

180 staff education. In our case series of 232 patients [4], eight patients (3\%) were excluded

181 because of CR early complications, including one lethal: anal incontinence, $n=2$; ischaemic

182 colitis, $n=1$; newly developed fistulas, $n=3$; cancerous colic stenosis, $n=1$; letal mesenterical

183 infarction: $\mathrm{n}=1$. Wu et al reported diarrhoea, vomiting, nausea, abdominal pain, and

184 abdominal distension that could affect until 14\% of patients, but were all relieved later during

185 CR course [6]. In other case series [4,5], the prevalence of CR-related technical problems (e.g.

186 tube disinsertions or obstructions, chyme leaks, stoma care problems,...) and gastrointestinal

187 side effects was not collected. In our experience, the most frequent side effects of CR are: the

188 difficulty to accept the food constraints of ingesting smooth puree meals that could impact on

189 mood, quality of life, and nutritional status; obstruction or displacement of the CR tube;

190 spasmodic pains during the resumption of normal downstream intestine function; severe

191 constipation, especially if antidiarrheal drugs are used (this is the reason why loperamide has

192 to be stopped before CR initiation).

193 One considerable advantage of CR is, by using the whole remnant small bowel, to prefigure

194 the post-SRIC intestinal function. Indeed some symptoms occurring during CR, such as pain,

195 fecal incontinence, diarrhoea, would have occurred after SRIC. Thus CR should allow

196 anticipating and even preventing situations that would have occurred after the SRIC with

197 potentially more serious consequences and sometimes would have required a second surgery.

198 For example, in case of fecal incontinence, anal biofeedback could be prescribed before the

199 SRIC. 


\section{Feasibility of home CR}

In 59 (28\%) patients of our French case series [4], CR was feasible at home in selected patients after specific training and education, where they must have acquired total autonomy for CR and basic stoma care. The median duration of home CR was $36 \pm 40$ days, accumulating 7.4 patients-years. For home CR, we have elaborated a dedicated clinical pathway including a thesaurus of solutions facing well defined technical problems. As a result, no patient had to stop CR, only a few were readmitted for minor problems, and none had to go back to PN. Unfortunately, at this time, home CR is not yet recognized by health insurances as a nutrition support technique. More studies, noteworthy multicentric, are needed to demonstrate safety and clinical benefits of home CR.

\section{Medicoeconomics benefits of CR}

At this time, PN remains the gold standard therapy until the patients underwent the SRIC. PN costs are much higher than those of EN and increase with complications [1]. The quick PN weaning with CR could have avoided 26.6 patients-years with PN [4]. As a great part of the costs of type 2 IF patients' therapies resulted from PN-related complications [11], CR could be associated with substantial cost-savings. The prospective randomized controlled trial FRY, supported by the French National Clinical Research Program, will determine the impact of CR compared to PN on the incidence of complications, healthcare costs and quality of life in IF patients with temporary high-output double enterostomy until a follow up of one month post-SIRC. Thanks to an additional ESPEN Technology grant 2015, the FRY trial will allow determining feasibility of home CR in a multicentric setting.

\section{Hypotheses upon the mechanisms of the clinical benefits of CR and enteroclysis}

In case of small bowel disruption, the absence of the ileal brake results in a non-inhibition of gastric emptying, gastric hypersecretion and accelerated intestinal motility, contributing to 
227 impaired absorption. Supposed mechanisms of CR-induced improvement of intestinal

228 function are the reabsorption of digestive secretions (gastric, pancreatic, and biliary succus)

229 and the restoration of the ileal brake. The latter was first suggested by Levy et al [12] who

230 showed that proximal stoma output was brake down by hydration solution enteroclysis into

231 the downstream small bowel (-20\%) and mainly by CR (-33\%). Plasma citrulline

232 normalization is the result of the increase in the functional enterocyte mass and of the better

233 extraction of intraluminal glutamine [7]. In case of terminal ileum resection, the enterohepatic

234 cycle of bile acids is disrupted leading to bile salts malabsorption. This results in an increase

235 in bile acid synthesis by the liver which, in turn, enhances hepatic lipogenesis and intrahepatic

236 cholestasis, resulting in plasma liver tests abnormalities. At physiological state, bile salts

237 activate the intracellular receptor FXR in the small intestine and liver epithelium [13]. FXR

238 activation is in part mediated by endocrine-acting fibroblast growth factor (FGF) 19, a bile

239 salt-induced enterokine. The release of the FGF19 subsequently inhibits bile salt synthesis

240 from cholesterol. In case of IF-induced enterohepatic cycle disruption, bile salts synthesis is

241 not inhibited, resulting in overproduction and liver accumulation that could have a direct

242 toxicity on hepatocytes. CR could act by restoring bile salts enterohepatic cycle and bile salts

243 signalling, decreasing liver inflammation and plasma liver tests. A study is ongoing to

244 demonstrate this hypothesis. Other mechanisms such as changes in intestinal microflora or 245 prevention of bacterial overgrowth deserve further investigation.

247 Conclusion

248 CR or enteroclysis are safe and easy-to-use nutrition support techniques. In case of IF

249 secondary to high output temporary enterostomy or ECF, CR is an efficient and reliable

250 technique of EN which corrects IF by restoring intestinal absorption. CR contributes to

251 improve nutritional status and to reduce plasma liver tests abnormalities, and is feasible at

252 home in well selected patients. By allowing the PN weaning within a short period, CR could 
253 be associated with substantial cost-savings. The prospective randomized controlled trial FRY, 254 supported by the French National Clinical Research Program, will determine the impact of 255 CR compared to PN on the incidence of complications, healthcare costs and quality of life in 256 IF patients with temporary high-output double enterostomy. This review may contribute to 257 improve the awareness of intensivists, digestive surgeons and gastroenterologists involved in 258 IF management to spread the use of CR and enteroclysis. 


\section{Key points}

261 - In case of intestinal failure secondary to high output temporary enterostomy or

262 enterocutaneous fistula, chyme reinfusion is an efficient and reliable technique of enteral

263 nutrition which corrects intestinal failure by restoring intestinal absorption, allowing

264 parenteral nutrition weaning in almost all patients.

265 - Chyme reinfusion contributes to improve nutritional status and to reduce plasma liver tests

266 abnormalities, and is feasible at home in well selected patients.

267 - Chyme reinfusion allows preparing efferent small bowel and colon to the surgical

268 reestablishment of intestinal continuity.

269 - In patients with exposed efferent small bowel, enteroclysis of hydration and/or enteral

270 nutrition solutions could allow improving hydration and nutritional status, as well as reducing

271 parenteral nutrition needs.

272 - Multicentre prospective randomized controlled trials are needed to determine the impact of

273 chyme reinfusion compared to parenteral nutrition on the incidence of complications,

274 healthcare costs and quality of life, as well as feasibility of home chyme reinfusion, in

275 intestinal failure patients with temporary high-output double enterostomy or enterocutaneous

276 fistula.

277

278 Acknowledgement

279 None

280

281 Financial support and sponsorship

282 None

283

284 Conflicts of interest 
285 RT declares no conflict of interest regarding this article. DP declares advisory activities

286 without any financial retribution with Labodial, Les Clayes Sous Bois, France. 


\section{References}

290 [1] Dibb M, Teubner A, Theis V, et al. Review article: the management of long-term parenteral nutrition. Aliment Pharmacol Ther 2013;37:587-603

[2] Pironi L, Arends J, Baxter J, et al. The Home Artificial Nutrition \& Chronic Intestinal

Failure and the Acute Intestinal Failure Special Interest Groups of ESPEN. ESPEN endorsed

294 recommendations. Definition and classification of intestinal failure in adults. Clin Nutr $295 \quad 2015 ; 34: 171-180$

** An important position paper written by experts from the European Society for Clinical

Nutrition and Metabolism (ESPEN) defining intestinal failure and proposed the use of chyme reinfusion and enteroclysis in type 2 intestinal failure patients.

299 [3] Pironi L, Arends J, Bozzetti F, et al. ESPEN guidelines on chronic intestinal failure in 300 adults. Clin Nutr 2016;35:247-307

301 [4] Picot D, Layec S, Dussaulx L, et al. Chyme reinfusion in patients with intestinal failure 302 due to temporary double enterostomy: a 15-year prospective cohort in a referral centre. Clin 303 Nutr 2016, in press

$304 * *$ This is the largest published case series of chyme reinfusion in intestinal failure patients 305 with temporary double enterostomy or enterocutaneous fistula. This study demonstrates the 306 improvement in intestinal absorption, nutritional status, and plasma liver tests abnormalities.

307 [5] Coetzee E, Rahim Z, Boutall A, Goldberg P. Refeeding enteroclysis as an alternative to 308 parenteral nutrition for enteric fistula. Colorectal Dis 2014;16:823-30

309 * One of the rare paper with [4] showing that chyme reinfusion allows adequate nutrition, 310 water and electrolyte balance, without resorting to parenteral infusions.

311 [6] Wu Y, Ren J, Wang G, et al. Fistuloclysis improves liver function and nutritional status 312 inpatients with high-output upper enteric fistula. Gastroenterol Res Pract 2014;2014:941514 
$313 *$ This study shows that enteroclysis improved plasma liver tests and nutritional status in

314 patients with high-output enterocutaneous fistula, particularly in biliary fistula patients.

315 [7] Picot D, Garin L, Trivin F, et al. Plasma citrulline is a marker of absorptive small bowel 316 length in patients with transient enterostomy and acute intestinal failure. Clin Nutr $317 \quad 2010 ; 29: 235-242$

318 [8] Calicis B, Parc Y, Caplin S, et al. Treatment of postoperative peritonitis of small-bowel 319 origin with continuous enteral nutrition and succus entericus reinfusion. Arch Surg $320 \quad 2002 ; 137: 296-300$

321 [9] Drenckpohl D, Vegunta R, Knaub L, et al. Reinfusion of succus entericus into the mucous 322 fistula decreases dependence on parenteral nutrition in neonates. ICAN: Infant, Child, \& 323 Adolescent Nutrition 2012;4:168-174

324 [10] Teubner A, Morrison K, Ravishankar HR, et al. Fistuloclysis can successfully replace 325 parenteral feeding in the nutritional support of patients with enterocutaneous fistula. Br J Surg 2004;91:625-631

327 [11] Saunders J, Parsons C, King A, et al. The financial cost of managing patients with type 2 328 intestinal failure; experience from a regional centre. e-SPEN Journal 2013;8:e80-e85

329 [12] Lévy E, Palmer DL, Frileux P, et al. Inhibition of upper reinfusion of succus entericus 330 into the distal small bowel: a clinical study of 30 patients with peritonitis and temporary enterostomy. Ann Surg 1983;198:596-600

332 [13] van Erpecum KJ, Schaap FG. Intestinal failure to produce FGF19: A culprit in intestinal 333 failure-associated liver disease? J Hepatol 2015;62:1231-3

$334 *$ A short editorial suggesting that intestinal failure-associated liver disease is not related only 335 to parenteral nutrition, but that bile acids signaling through the FXR-FGF19 axis, should be involved. 


\section{Figure legends}

339 Figure 1 - Example of a temporary double enterostomy. The small bowel continuity is

340 disrupted with two small bowel segments exposed to the abdominal wall: the upstream

341 afferent segment, with impaired digestive and absorptive function, and a downstream efferent

342 segment, totally deprived of digestive secretions, bowel flow and succus entericus. The

343 feeding tube is inserted in the downstream small bowel, and is ready for enteroclysis or 344 chyme reinfusion.

Figure 2 - Chyme reinfusion technique with the automated pump Enteromate $I^{\circledR}$ (A) and the portable Enteromate ${ }^{\circledR}$ Mobile (B) (Labodial, Clayes-sous-Bois, France). (A) The

348 left pump works permanently and aspirates the jejunal effluent from the upstream small bowel 349 afferent stoma toward a $30 \mathrm{ml}$ disposable plastic container, which is hung on an electronic 350 steelyard. The upper stoma pouch is always empty. The weight of the container is continuously and electronically monitored. When the minimal volume of approximately $10 \mathrm{ml}$ is exceeded, the second pump starts and the contents are infused into the diverted downstream small bowel until the return to minimal volume. (B) Once the patient has been adequately trained and is capable of correctly adjusting the rate of reinfusion, portable non-autoregulated Enteromate Mobile ${ }^{\circledR}$ pump was used secondly to give autonomy to the patient during the hospitalization and at home. This pump is autonomous thanks to batteries. 
Figure 1

359

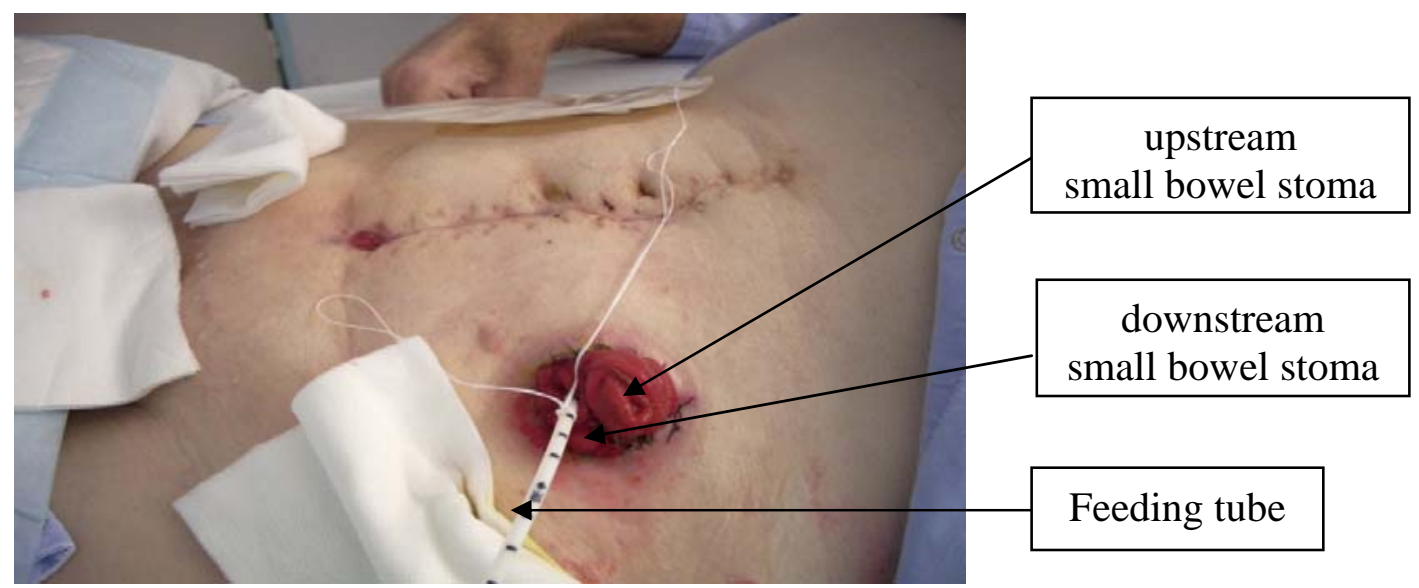

361

362 
Figure 2

364

A

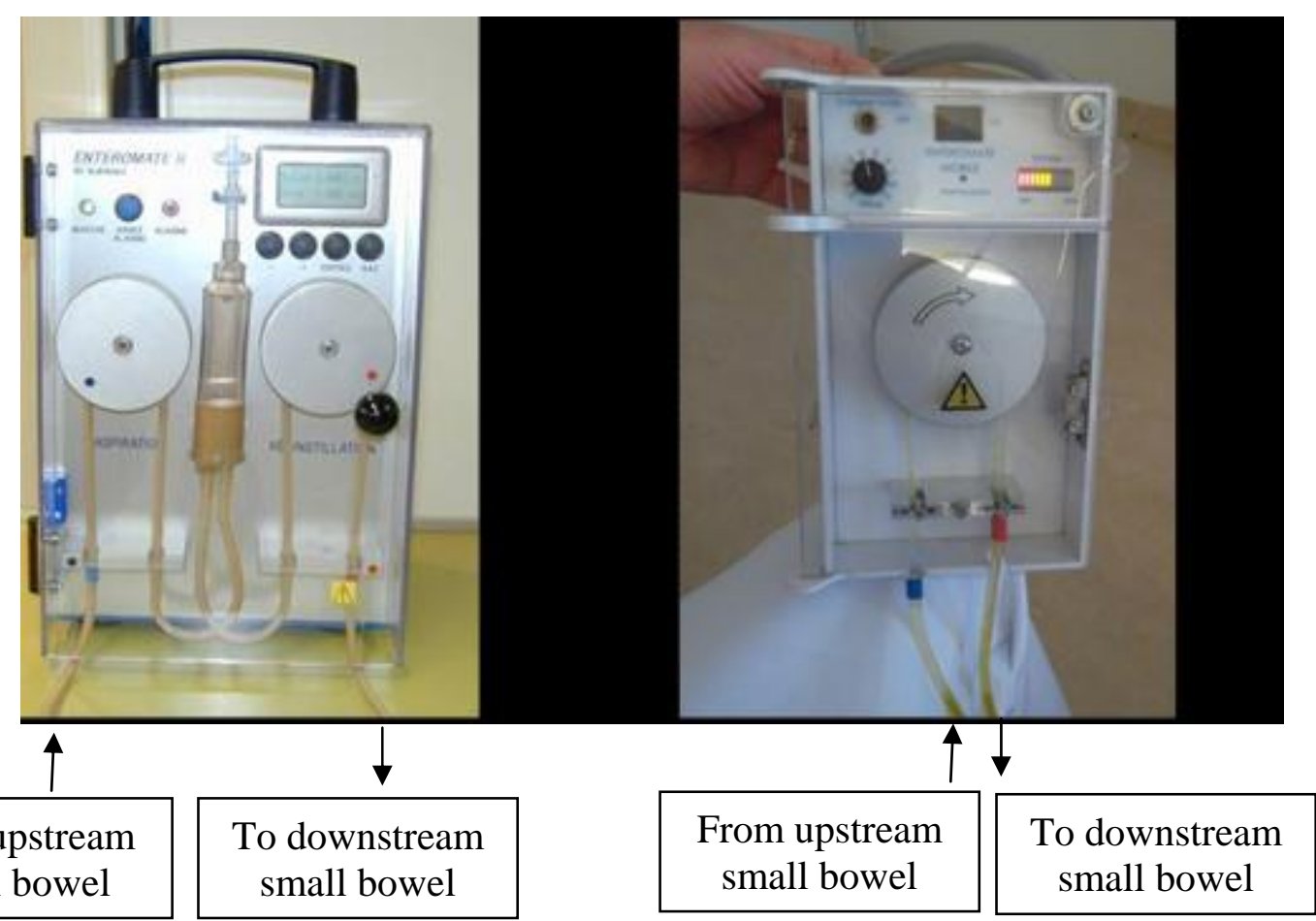

368 